\title{
Experiências e sentimentos de mães diante da doença de Moyamoya de seus filhos
}

\author{
Experiences and feelings of mothers of children with Moyamoya disease
}

\author{
Daniela Bravalhieri da SILVA' \\ Paula de Marchi Scarpin HAGEMANN ${ }^{1}$ \\ Camila PEREIRA ${ }^{1}$ \\ Carmen Maria Bueno NEME ${ }^{1}$
}

\begin{abstract}
Resumo
A doença de Moyamoya é uma grave enfermidade crônica, caracterizada por oclusão das grandes artérias na base do cérebro e é considerada importante causa de acidente vascular cerebral. O objetivo deste estudo consistiu em investigar a experiência e os sentimentos de mães diante do diagnóstico e da evolução da doença de Moyamoya e de seu tratamento em seus filhos. Trata-se de uma pesquisa descritiva, com análises quantitativa e qualitativa. Participaram do estudo oito mães de filhos com diagnósticos da doença de Moyamoya provenientes de três estados da região Sudeste do Brasil. Os dados foram coletados por meio de questionários via página da Internet. Os resultados mostram que o processo de diagnóstico da doença foi vivido com temores e tristeza, agravados pelas dificuldades diagnósticas e escassez de informações. As mães relataram desespero, medo, dificuldade de aceitação, culpa e temor na realização de angiografia cerebral e cirurgia. A fé e a espiritualidade foram referidas como fontes de apoio e de manutenção da esperança. Assinala-se a necessidade de estudos que colaborem para maior esclarecimento sobre a doença e a vivência de doentes e familiares.
\end{abstract}

Palavras-chave: Doença de Moyamoya; Estados emocionais; Mães; Terapêutica.

\begin{abstract}
Moyamoya disease is as a serious chronic disease characterized by an occlusion of the main arteries of the brain, and it is considered an important cause of stroke. The purpose of this study was to investigate the experiences and feelings of mothers facing their child's diagnosis, progress, and treatment of Moyamoya disease. This is a descriptive study with quantitative and qualitative analyses. Eight mothers of children with Moyamoya disease from three different southeastern states in Brazil participated in the study. Data were collected via Internet questionnaires. The results show that fear and sadness pervaded the mothers' experiences of the disease diagnosis, which was aggravated by the scarcity of information available. Mothers reported feeling despair, anxiety, guilt, difficulty of acceptance, and fear related to cerebral angiography and brain surgery. Faith and spirituality were referred to as sources of support and maintenance of hope. There is a need for further studies that can bring greater clarity about this disease and the experience of patients and their families.
\end{abstract}

Keywords: Moyamoya disease; Emotional states; Mothers; Therapeutics.

${ }^{1}$ Universidade Estadual Paulista Júlio de Mesquita Filho, Faculdade de Ciências, Departamento de Psicologia. Av. Engenheiro Luis Edmundo Coube, s/n., 17033-360, Bauru, SP, Brasil. Correspondência para/Correspondence to: P.M.S. HAGEMANN. E-mail: <pmscarpin@gmail.com>. 
A doença de Moyamoya (DMM), ou doença cerebrovascular oclusiva, é uma desordem vaso-oclusiva de etiologia desconhecida, caracterizada como doença idiopática, estenótica e progressiva. Nos exames de angiografia cerebral, a circulação sanguínea colateral aparece com aspecto nebuloso, descrito na literatura como "nuvem de fumaça". Essa aparência foi responsável pelo uso da palavra "Moyamoya", em 1957, no Japão. É uma doença rara e considerada importante causa de Acidente Vascular Cerebral (AVC) (Matushita, Matushita, Cardeal, \& Andrade, 2014; Nascimento et al., 2011; Ruiz \& Pulgarin; 2012).

Embora relatada com maior frequência na população da Ásia Oriental, tem sido observada crescente incidência na população americana e europeia e em indivíduos de outras etnias (Matushita et al., 2014; Nascimento et al., 2011). Apesar de um recente estudo genético ter identificado um gene de susceptibilidade possível, a etiologia da DMM ainda não foi totalmente definida (Fujimura et al., 2014).

Existem dois picos de incidência da patologia relatados na literatura, um em torno de 10 anos de idade e outro entre 30 e 40 anos, sendo mais comum a ocorrência tardia em mulheres do que em homens (Kim, 2016; Ruiz \& Pulgarin, 2012). O diagnóstico de DMM deve ser considerado, principalmente, quando ocorrem sintomas entre crianças com menos de três anos de idade e entre jovens, dada a maior gravidade nestes casos (Matushita et al., 2014; Shoukat, Itrat, Taqui, Zaidi, \& Kamal, 2009).

A doença de Moyamoya apresenta associação com diversas patologias, sendo que a síndrome de Down tem sido sistematicamente relatada como a ela associada (Junqueira \& Moura-Ribeiro, 2002; Matushita et al., 2014). A prevalência de síndrome de Down em pacientes com DMM é 26 vezes maior, em comparação com sua prevalência entre nascidos vivos sem DMM (Kainth, Chaudhry, Kainth, Suri, \& Qureshi, 2013). Clinicamente, os sintomas da DMM podem variar muito de um caso para outro. As anormalidades irão ocorrer de acordo com a idade do paciente durante as primeiras manifestações, como eventos cerebrovasculares isquêmicos, ataque isquêmico transitório, tipicamente mais prevalente em crianças, e eventos hemorrágicos, mais comuns em adultos. As pessoas com a doença descobrem comumente sua presença após o AVC, que se constitui um dos principais sintomas e pode deixar sequelas temporárias ou permanentes (Nascimento et al., 2011; Ruiz \& Pulgarin, 2012).

Dentre as alternativas indicadas para o tratamento, a cirurgia de revascularização cerebral é a que tem obtido os melhores resultados e tem se mostrado um tratamento eficaz para prevenir AVC isquêmico em pacientes com a forma isquêmica da doença (Kim et al., 2015; Kim, Oh, Bang, Kim \& Cho, 2016). Apesar de ainda controversa, a realização de revascularização para prevenir hemorragias é ressaltada na literatura (Miyamoto et al., 2014), inclusive como meio eficiente para reverter parâmetros hemodinâmicos pré-operatórios, prevenir a deterioração neurológica e melhorar a qualidade de vida a longo prazo (Matushita et al., 2014).

Portadores da DMM, especialmente os que têm AVC na forma hemorrágica, apresentam prejuízo significativo da qualidade de vida (Su, Haj, Zhang, Wu, \& Yu, 2013). Além disso, o comprometimento neurocognitivo constitui-se preocupação frequente. A ocorrência de sequelas pós-AVC pode exigir avaliações neurológicas e neuropsicológicas, bem como tratamentos especializados (Nascimento et al., 2011). Em adultos, as alterações neuropsicológicas são consideradas devido ao envolvimento frequente da área frontotemporal e à predominância de alterações na substância branca, podendo resultar no desenvolvimento de disfunções cognitivas (Nakamizo, Kikkawa, Hiwatashi, Matsushima, \& Sasaki, 2014; Kazumata et al., 2015). Em crianças, essas alterações são frequentes, apesar do funcionamento intelectual geral se apresentar normal (Hsu, Kuo, Hua, \& Yang, 2014).

Famílias com filhos afetados por fatores genéticos que possam envolver sequelas funcionais, estruturais e psicológicas são vulneráveis a diferentes graus de estresse (Tabaquim \& Marquesini, 2013). A exposição a uma doença crônica grave pode dificultar o manejo de condições agudas, comprometendo a qualidade de vida tanto dos doentes quanto dos 
familiares (Neme, 2012). Santos e Cardoso (2015) identificaram altos índices de estresse em familiares cuidadores de pessoas em sofrimento psíquico crônico. Instala-se, assim, uma vida de dependência ligada à doença, com importantes desgastes para o doente e a família (Furtado \& Lima, 2003). As mães costumam ser as principais cuidadoras de filhos doentes e hospitalizados, sendo que a falta de apoio de outros membros da família pode levar o cuidador principal a desenvolver sentimentos de solidão e abandono (Beuter, Brondani, Szareski, Cordeiro, \& Roso, 2012).

Mães cuidadoras de filhos hospitalizados com piora do quadro clínico ou em fase de definição diagnóstica tendem a utilizar estratégias de enfrentamento particulares para suportar a situação, destacando-se o apego à fé religiosa (Beuter et al., 2012; Costa, Mombelli, \& Marcon, 2009; Rooke \& Pereira-Silva, 2016). Estudo realizado por Horta, Neme, Capote e Gibran (2003) mostrou a importância dos aspectos religiosos e espirituais no processo de enfrentamento e reabilitação de doenças graves, especialmente nas condições crônicas.

O enfrentamento religioso tem sido estudado no contexto das práticas em saúde, destacando-se a utilização de estratégias cognitivas e/ou comportamentais ligadas a crenças religiosas pessoais para lidar com os estressores (Faria \& Seidl, 2005). A religiosidade e a espiritualidade têm demonstrado grande efeito sobre a saúde física (Fornazari \& Ferreira, 2010) e mental (Oliveira \& Junges, 2012), além de se mostrarem importantes recursos de enfrentamento, especialmente quando há pouco controle sobre a gênese e a evolução de uma doença grave (Horta et al., 2003). Dessa forma, a espiritualidade constitui-se importante recurso para lidar com situações de crise que exigem uma melhor adaptação (Colesante, Gomes, Morais, \& Collet, 2012).

O diagnóstico de uma doença grave instaura uma situação de crise, especialmente quando pode levar à morte iminente ou, até mesmo, por sua cronicidade, exigir tratamentos invasivos e de longa duração, alterando toda a dinâmica familiar (Neme, 2012). Tendo em vista a gravidade da DMM e a escassez de pesquisas no campo da Psicologia sobre a doença, este estudo visou investigar a experiência e os sentimentos de mães diante do diagnóstico, evolução da doença e seu tratamento em seus filhos, bem como os efeitos desta trajetória no funcionamento familiar.

\section{Método}

\section{Participantes}

Participaram do estudo oito mães de filhos diagnosticados com DMM, contatadas por meio de uma página da Internet criada com o objetivo de reunir pessoas acometidas pela DMM e seus familiares. O número de participantes representou a totalidade (100\%) de contatos que foi possível realizar através do referido site. O critério de inclusão na amostra foi ser mãe de filho diagnosticado com a DMM. Trata-se de uma amostra de conveniência não probabilística, o que faz com que os dados coletados não sejam representativos para a totalidade da população.

\section{Instrumentos}

Foram elaborados três questionários com questões semiestruturadas visando obter as informações pretendidas. No questionário A, constaram sete perguntas relativas aos dados pessoais e demográficos da mãe e do(a) filho(a), além de informes sobre a família. Por meio do questionário $B$, foram investigados cinco itens sobre a doença e os tratamentos e, pelo questionário $C$, com 26 questões, investigou-se as experiências e sentimentos maternos frente à doença e ao tratamento de seus filhos.

\section{Procedimentos}

Participaram do estudo todas as mães contatadas através da página da Internet. 
Inicialmente, elas foram esclarecidas quanto aos objetivos da pesquisa e convidadas a colaborar. Informações gerais foram fornecidas, a princípio, pela Internet e, posteriormente, por meio do Termo de Consentimento Livre e Esclarecido (TCLE), enviado pelo correio após concordarem em participar. Os questionários para a coleta de dados foram enviados por correio eletrônico, com prazo de 30 dias para sua devolução, o que foi cumprido por todas as participantes.

Para a análise dos dados, os questionários respondidos pelas mães foram lidos separadamente, buscando-se compreender cada caso. A seguir, foi realizada leitura atenta das respostas a cada uma das questões, a fim de se obter os dados do conjunto. Posteriormente, as respostas foram analisadas, descritas e organizadas em cinco grandes grupos: dados demográficos, familiares e referentes ao adoecimento; a vivência do diagnóstico; o suporte emocional obtido via troca de experiências; o vivenciar da hospitalização e da cirurgia; e religiosidade como fonte de conforto e apoio.

Todos os procedimentos seguidos no estudo estão de acordo com os padrões éticos estabelecidos pela Resolução Normativa 196/96 do Conselho Nacional de Saúde que regulamenta pesquisas envolvendo seres humanos (Conselho Nacional de Saúde, 1996). O estudo se iniciou após sua aprovação pelo Comitê de Ética em Pesquisa da Faculdade de Ciências de Bauru, da Universidade Estadual Paulista (Processo n 716/46/01/09).

\section{Resultados e Discussão}

\section{Dados demográficos, familiares e referentes ao adoecimento}

Cinco das participantes residiam no Estado de São Paulo, duas no Estado de Minas Gerais e uma no Espírito Santo. As famílias eram constituídas, em média, por quatro pessoas (casal e um a três filhos). A Tabela 1 apresenta os dados referentes ao gênero e idade dos filhos com DMM, além de informações sobre o adoecimento.

Tabela 1

Dados dos filhos com doença de Moyamoya e do adoecimento

\begin{tabular}{|c|c|c|c|c|c|c|}
\hline Mães & $\begin{array}{l}\text { Sexo e idade } \\
\text { dos filhos }\end{array}$ & Comorbidades & $\begin{array}{l}\text { Idade dos primeiros } \\
\text { sintomas }\end{array}$ & $\begin{array}{l}\text { AVC? } \\
\text { Tipo }\end{array}$ & $\begin{array}{l}\text { Tratamento e } \\
\text { reabilitação }\end{array}$ & $\begin{array}{l}\text { Cefaleias/ } \\
\text { convulsões }\end{array}$ \\
\hline$A$ & $\begin{array}{l}\text { Feminino, } \\
19 \text { anos }\end{array}$ & $\begin{array}{c}\text { Paralisia } \\
\text { cerebral leve }\end{array}$ & 10 anos & $\begin{array}{l}\text { Sim, } \\
\text { Isquêmico }\end{array}$ & $\begin{array}{l}\text { Fonoaudiologia, } \\
\text { Fisioterapia e } \\
\text { Hidroterapia }\end{array}$ & Sim \\
\hline B & $\begin{array}{l}\text { Masculino, } \\
11 \text { anos }\end{array}$ & Não & 6 anos & $\begin{array}{l}\text { Sim, } \\
\text { Isquêmico }\end{array}$ & Fisioterapia & Sim \\
\hline C & $\begin{array}{l}\text { Feminino, } \\
10 \text { anos }\end{array}$ & $\begin{array}{l}\text { Tireoidite de } \\
\text { Hashiniro }\end{array}$ & 6 anos & $\begin{array}{l}\text { Sim, } \\
\text { Isquêmico }\end{array}$ & Não & Não \\
\hline D & $\begin{array}{l}\text { Feminino, } \\
10 \text { anos }\end{array}$ & $\begin{array}{l}\text { Síndrome de } \\
\text { Down }\end{array}$ & 7 anos & $\begin{array}{l}\text { Sim, } \\
\text { Isquêmico }\end{array}$ & Fisioterapia & Sim \\
\hline $\mathrm{E}$ & $\begin{array}{l}\text { Masculino, } \\
30 \text { anos }\end{array}$ & $\begin{array}{l}\text { Hérnia de } \\
\text { disco }\end{array}$ & 22 anos & $\begin{array}{l}\text { Sim, Hemor- } \\
\text { rágico } \\
\text { e Isquêmico }\end{array}$ & $\begin{array}{l}\text { Fonoaudiologia, } \\
\text { Fisioterapia, } \\
\text { Terapia ocupacional }\end{array}$ & Sim \\
\hline $\mathrm{F}$ & $\begin{array}{l}\text { Feminino, } \\
26 \text { anos }\end{array}$ & Não & 22 anos & $\begin{array}{l}\text { Sim, } \\
\text { Isquêmico }\end{array}$ & Não & Sim \\
\hline G & $\begin{array}{l}\text { Feminino, } \\
28 \text { anos }\end{array}$ & Não & 19 anos & $\begin{array}{l}\text { Sim, } \\
\text { Isquêmico }\end{array}$ & Fisioterapia & Sim \\
\hline $\mathrm{H}$ & $\begin{array}{l}\text { Masculino, } \\
6 \text { anos }\end{array}$ & $\begin{array}{l}\text { Síndrome de } \\
\text { Down }\end{array}$ & 5 anos & $\begin{array}{l}\text { Sim, } \\
\text { Isquêmico }\end{array}$ & $\begin{array}{l}\text { Fisioterapia e } \\
\text { Fonoaudiologia }\end{array}$ & $\operatorname{sim}$ \\
\hline
\end{tabular}

Nota: AVC: Acidente Vascular Cerebral.

Observou-se maior ocorrência da DMM no sexo feminino (cinco casos) do que no masculino (três casos), corroborando os dados de Ruiz e Pulgarin (2012) e Kim (2016), que pontuam frequência da doença ligeiramente maior em mulheres. A idade atual dos filhos situou-se na faixa de 19 a 30 anos entre os adultos e de 6 a 11 anos entre as crianças. Os primeiros sintomas da doença apareceram na infância 
em cinco casos e na idade adulta nos demais. Em todos os casos ocorreu o AVC isquêmico, sendo que em um deles (filho da mãe E), no qual os primeiros sintomas surgiram aos 22 anos, ocorreu também o AVC hemorrágico. De acordo com Nascimento et al. (2011), os adultos tendem a apresentar a forma hemorrágica da doença mais frequentemente. Neste estudo, cinco dos oito casos apresentaram os primeiros sintomas na primeira década de vida, dois na terceira e um caso na segunda década, o que reforça as indicações de Nascimento et al. (2011), Ruiz e Pulgarin (2012) e Kim (2016) quanto à variabilidade da idade em que surge a patologia.

Entre os filhos das mães participantes, apenas dois tinham descendência oriental. Embora a maior ocorrência da DMM seja entre japoneses, há relatos crescentes de sua ocorrência em outras etnias (Matushita et al., 2014; Nascimento et al., 2011; Ruiz \& Pulgarin, 2012). Apenas três casos não apresentavam comorbidades ou diagnósticos associados e, entre os cinco restantes, dois tinham síndrome de Down, o que vai ao encontro dos resultados das pesquisas de Junqueira e MouraRibeiro (2002), de Kainth et al. (2013) e de Matushita et al. (2014).

No que se refere à ocorrência de AVC, três mães responderam que seus filhos tiveram sequelas temporárias e permanentes, duas, que tiveram sequelas permanentes e três, sequelas temporárias. Sintomas como cefaleias e crises convulsivas não ocorreram em apenas um caso (mãe C), corroborando resultados de estudos referindo a presença destes sintomas entre pacientes com DMM (Ruiz \& Pulgarin, 2012).

Excetuando-se uma menina de 10 anos de idade (mãe C), os demais casos precisaram de assistência especializada (fisioterapia, fonoaudiologia, hidroterapia e terapia ocupacional). A necessidade de assistência especializada pós-AVC é mencionada na literatura (Hsu et al., 2014; Nakamizo et al., 2014; Nascimento et al., 2011). A família necessita estar preparada para cuidar do paciente e modificar suas rotinas, o que foi referido pelas mães. Diante dessa realidade, a necessidade de reorganização de papéis torna-se inevitável, o que pode levar a profundas repercussões na dinâmica familiar (Nóbrega, Reichert, Silva, Coutinho, \& Collet, 2012).

Segundo relato das participantes, a dedicação ao filho diagnosticado com a doença modificou a rotina familiar, dificultando, muitas vezes, o relacionamento com os outros filhos, que passaram a se queixar por se sentirem abandonados. A DMM, assim como toda doença crônica, configura-se como importante agente estressor, podendo atingir negativamente o desenvolvimento normal da criança e as relações do sistema familiar. Além disso, o possível despreparo psicológico da família em relação ao enfrentamento de uma condição crônica exerce impacto desfavorável na adaptação da criança e da família à nova situação, o que pode levar a prejuízos na qualidade de vida de ambos (Neme, 2012; Nóbrega et al., 2012; Silva, Weiss, Bernardes, \& Souza, 2006). Em decorrência dos sintomas da patologia, das sequelas do AVC e do tratamento, seis mães ressaltaram que mudanças na rotina familiar foram invitáveis. Silva et al. (2006) destacam que as alterações emocionais e sociais que se instalam em toda a família exigem cuidados adaptativos. Neme (2012) indica a necessidade de se desenvolver mecanismos de proteção, capazes de diminuir possíveis danos decorrentes dos riscos representados pela doença crônica, tanto para o doente quanto para a família.

\section{A vivência do diagnóstico}

A falta de um diagnóstico inicial preciso levou as mães a temerem piores possibilidades. A preocupação e o desespero foram sentimentos frequentes e a espera pelo diagnóstico foi referida como uma experiência de impotência e extrema angústia, sendo que os sentimentos de impotência são vivências comuns para mães de filhos doentes (Santos \& Gonçalves, 2008).

Excertos das respostas de duas mães exemplificam a dimensão do sofrimento gerado pela incerteza quanto ao diagnóstico. 
Fiquei sem saber o que estava acontecendo. No primeiro episódio ela foi logo hospitalizada e ficou boa logo, não diagnosticaram o que Ihe tinha acontecido. Somente pediram para passá-la em vários especialistas e vários exames estavam sendo feitos. No segundo, ela teve uma convulsão forte. Em 24 horas passei por dois hospitais e ela sempre toda mole, os médicos achavam que era somente pela convulsão e por ser portadora de paralisia cerebral (Mãe A).

Sentimento de tristeza, de dor, de desespero, de que o mundo havia acabado, mas ao mesmo tempo tive que mostrar que era uma fortaleza (Mãe E).

A demora em obter resultados dos exames soma-se a outros fatores desencadeantes do medo: intranquilidade e nervosismo. Essas vivências se evidenciam quando se percebe o risco de morte. Assim como o medo, a insegurança manifestada pela mãe é relacionada à dúvida quanto ao diagnóstico e à falta de informação (Ribeiro, 1999).

A angiografia cerebral é o principal exame para o diagnóstico da doença. Por se tratar de um procedimento invasivo e decisivo para o diagnóstico, este foi um momento vivido com grande tensão, apreensão e angústia pelas participantes. A realização de procedimentos invasivos causa grande impacto no familiar, podendo levar a sentimentos de apreensão, impotência e desespero (Beuter et al., 2012). Além disso, a falta de um diagnóstico claro e de informações mais precisas dificultam um enfrentamento mais efetivo (Neme, 2005). Situações como esta, permeada de riscos, geram ansiedades e temores, como referido nas falas: "Tensão, preocupação e expectativa de resultado" (Mãe G); "Medo e muita insegurança" (Mãe H).

Como mencionado anteriormente, a descoberta de que um filho tem uma doença crônica pode gerar forte impacto emocional nas famílias, principalmente nas mães, que são as principais cuidadoras. Essa fase é tida como uma do desconhecido (Colesante et al., 2012). A reação das participantes do presente estudo confirma as colocações de Colesante et al. (2012), pois, no momento do diagnóstico, os sentimentos de angústia, temor e incerteza se intensificaram. "Me sentia perdida porque eu não sabia o que era; sabia que era sério, mas o médico dele não esclareceu nada para mim, então fiquei meio perdida, sozinha" (fala da mãe $H$ ).

Nos relatos, como o colocado acima, as mães revelam sentimentos de desamparo decorrentes da falta de informação. As que foram informadas de que se tratava de uma doença grave, rara e crônica, vivenciaram a notícia de modo impactante: "Foi um choque, fiquei muito assustada..." (fala da mãe H).

Na doença de Moyamoya, a busca por maiores informações acerca da doença e do tratamento constitui-se em causa de grande apreensão, principalmente por se tratar de uma mazela rara e de difícil diagnóstico. As incertezas e dificuldades para a obtenção do diagnóstico final foram vivenciadas por seis das mães participantes, evidenciando diagnósticos equivocados durante o processo de descoberta da patologia. Foram citados: trombofilia (mãe $H$ ), anemia falciforme (mãe B), epilepsia (mãe H) devido a paralisia cerebral (mãe $A$ ), enxaqueca com aura (mãe $C$ ), vasculite cerebral (mãe F) e herpes (mãe E). Apenas duas participantes (D e G) relataram a obtenção de um único diagnóstico. A dificuldade para a realização do mesmo relaciona-se à raridade da doença, conforme descrito por Nascimento et al. (2011) e Ruiz e Pulgarin (2012).

A confirmação do diagnóstico desencadeou reações negativas, aumentou a apreensão e causou surpresa e revolta. Del Bianco Faria e Cardoso (2010) identificaram em seus estudos que a descoberta de uma doença é vivenciada com muita surpresa pelos cuidadores e a confirmação de um diagnóstico grave parece ser gerador de muitas expectativas negativas.

Nos relatos das mães, identifica-se a tentativa de compreender o adoecimento de seus filhos. Crenças relacionadas ao castigo, à culpa ou à predestinação foram referidas, aumentando a revolta (mães $A$ e E), a impotência (mãe $G$ ) ou o 
inconformismo (mãe H). O sentimento de culpa é muito frequente, levando os pais a atribuírem a patologia a um castigo ou punição (Ribeiro, 1999). Del Bianco Faria e Cardoso (2010) chamam a atenção para a sobrecarga emocional, que tende a ser intensificada quando acrescida do sentimento de culpa. O relado da mãe $C$ exemplifica essa ocorrência: "A princípio [me senti] culpada, pois a descendência de japonês que meu filho tem é de minha parte, meu pai era japonês...".

\section{O suporte emocional obtido via troca de experiências}

A necessidade de maiores informações sobre a doença e seu prognóstico levou as mães a procurarem outras pessoas com DMM. Cinco participantes referiram dificuldades para encontrar pessoas com o mesmo diagnóstico, entretanto, todas conseguiram estabelecer contato entre si via página da Internet e duas delas também se comunicaram por telefone. Ao encontrarem pessoas nas mesmas condições, as mães relataram sentimentos de alívio, força e amparo, que possibilitaram o aumento da esperança e a tentativa de aceitação. Contudo, para a mãe $A$, a experiência foi diferente. A heterogeneidade dos casos que conheceu trouxe outras dúvidas e inseguranças, levando-a, aparentemente, a negar a característica de raridade da doença que acometeu sua filha aos 10 anos de idade. Disse ela: "Depois que conheci outras pessoas com Moyamoya, aí é que não entendi mesmo o porquê da doença. Sinceramente, acho que essa doença não é tão rara como falam" (mãe A).

O comportamento de negar o problema evidencia características importantes do funcionamento familiar, o que pode ser considerado como uma estratégia de enfrentamento negativa (Farinhas, Wendling, \& Dellazzana-Zanon, 2013). Costa et al. (2009) ressaltam que o objetivo do processo de enfrentamento é preservar a saúde mental, sendo que as estratégias defensivas caracterizam-se por ações adaptativas acionadas em situações penosas, com o objetivo de evitar o sofrimento mental. Neme e Lipp (2010) assinalam o caráter individual do processo de enfrentamento, visto que este é influenciado pela personalidade, vivências e necessidades individuais.

\section{O vivenciar da hospitalização e da cirurgia}

Ao responderem sobre sentimentos frente à hospitalização e cirurgia de seus filhos, as mães do presente estudo citaram o medo, a ansiedade e a angústia pela imprevisibilidade dos resultados. Silva et al. (2006) mostraram que a hospitalização em decorrência de doença grave pode ter dois desdobramentos: a cura e a morte. Os sentimentos de insegurança diante do futuro resultam em aumento do estresse, sendo a raiva e o ressentimento reações comuns diante da doença e da finitude. Esses sentimentos foram revelados pelas participantes ao relatarem o inconformismo e a revolta devido aos sintomas e à doença rara de seus filhos.

A ameaça de perda pode dificultar a ação das famílias ao lidarem com a situação de crise. Porém, os pais não costumam falar abertamente sobre essa possibilidade, vivendo solitariamente o sofrimento (Nieweglowski \& Moré, 2008). Esse sofrimento solitário foi citado por três mães cujos filhos apresentaram a doença na infância. Elas referiram que evitavam falar sobre o assunto, buscando manter a força e poupar a criança e os demais familiares.

A necessidade de cirurgia gerou sentimentos conflitantes nas mães. Todas referiram esperança com o procedimento e consciência da necessidade do mesmo, mas, para todas, apareceu o medo dos riscos que a cirurgia oferecia. Silva et al. (2006) ressaltam que a cirurgia é um novo desconhecido e que os familiares sentem-se inseguros quanto à sua evolução. As mães D e H relataram ter desmarcado a cirurgia dos filhos devido ao medo e insegurança.

Cinco mães com filhos menores de 18 anos tiveram que decidir se fariam ou não o 
procedimento, o que gerou intenso sofrimento e dificuldades para a tomada de decisão, conforme depoimento da mãe C: "Demorei um ano para decidir operar, resisti até o médico me explicar que não podíamos mais esperar..." (mãe C).

Para as demais mães, mesmo sabendo dos altos riscos de uma cirurgia cerebral, o desejo de resolver o problema auxiliou na tomada de decisão. "Diante de tudo o que passei, no momento em que ouvi do médico que tinha que operar, já estava preparada e aceitei porque queria meu filho salvo e ao meu lado" (mãe E). Ao serem questionadas sobre os conselhos que dariam a pais e familiares de pessoas com DMM, as participantes foram unânimes em aconselhar a realização da cirurgia, a despeito dos possíveis riscos.

\section{Religiosidade como fonte de conforto e apoio}

A análise das respostas demonstrou que a religiosidade foi importante no enfrentamento da doença. Quatro mães mencionaram que, ao encararem a patologia de seus filhos como provação, encontraram força na religião. Esse resultado está de acordo com dados da literatura (Beuter et al., 2012; Horta et al., 2003; Rooke \& Pereira-Silva, 2016), os quais mostram que a fé religiosa possibilitou a manutenção da esperança frente ao diagnóstico. A confiança na equipe de saúde, a superação de intercorrências e a possibilidade de encarar a vida de forma mais positiva foram facilitados pela fé e concepções religiosas. Os relatos das mães deste estudo mostram a importância do enfrentamento religioso, como ressaltam Faria e Seidl (2005).

Me senti uma escolhida de Deus, pois Deus sabia que eu ia cuidar, ajudar e lutar para salvar meu filho, ele acreditou em mim, assim como acredito nele (mãe C).

... É preciso ter muita força, pedir a Deus, porque é só Ele que nos sustenta e não desistir de lutar jamais, seja a dificuldade que for ... . Precisamos lutar até quando Deus quiser, pois Ele nos dá nossas cruzes para carregarmos, com amor. Em tudo Deus tem um propósito ... eu creio (mãe H).

\section{Considerações Finais}

Os resultados obtidos mostram que o processo de diagnóstico da DMM foi vivido com temor, desespero, ansiedade e tristeza pelas participantes. A falta de informações sobre a doença, as incertezas acerca dos tratamentos e os riscos da cirurgia foram fontes de estresse e sofrimento, desencadeando sentimentos de revolta, culpa, insegurança e medo.

Dado seu caráter crônico e progressivo, crianças e adultos portadores da DMM demandam atenção intensa e continuada, principalmente por parte das mães, as principais cuidadoras. 0 impacto da doença e do tratamento na dinâmica e funcionamento familiar foi ressaltado, revelando amplas necessidades adaptativas dos membros da família. Evidenciou-se, nos relatos das participantes, a necessidade de informações e de apoio emocional e social por meio da troca de experiências com outras famílias e pessoas com DMM, o que as levou a buscar contatos via Internet. As mães também ressaltaram o importante papel da fé e da religiosidade na aceitação do diagnóstico e do percurso de tratamento.

A despeito do reduzido número de participantes, os dados obtidos corroboram os da literatura, principalmente no que se refere às manifestações da doença, às dificuldades para obtenção de diagnósticos e informações sobre prognósticos e tratamentos, aos temores de exames e cirurgias, aos sentimentos e experiências das mães e aos impactos da doença na família. Embora não passíveis de generalização, os resultados aqui descritos são relevantes, tendo em vista seu caráter pioneiro no Brasil, especialmente no campo da Psicologia, podendo colaborar para alertar profissionais de saúde e a população acerca da DMM. 
Busca-se, dessa maneira, contribuir para a redução do subdiagnóstico da patologia e minimizar parte do sofrimento de pacientes, mães e familiares, carentes de informações e relatos de experiências. Sugere-se, assim, que a DMM, bem como seu impacto nos familiares sejam mais bem divulgados entre os profissionais de saúde, diminuindo o isolamento, a falta de informações e os riscos de um diagnóstico equivocado ou demorado. Destaca-se, também, a necessidade de estudos epidemiológicos que ampliem o conhecimento sobre a incidência e prevalência da doença no Brasil. É importante destacar, também, a necessidade de novos estudos que incluam a participação de outros membros da família, com foco em fatores relacionados ao estresse e à qualidade de vida e no papel dos profissionais de saúde, dentre os quais, o psicólogo, na fase diagnóstica e na trajetória de tratamentos.

\section{Colaboradores}

D.B. SILVA foi responsável pela concepção e desenho, P.M.S. HAGEMANN foi responsável pela análise e interpretação dos dados, discussão e revisão final. C. PEREIRA foi responsável pela discussão dos resultados, C.M.B. NEME foi responsável por orientar as alunas com relação aos aspectos metodológicos do estudo, bem como pela revisão e aprovação da versão final do artigo.

\section{Referências}

Beuter, M., Brondani, C. M., Szareski, C., Cordeiro, F. R., \& Roso, C. C. (2012). Sentimentos de familiares acompanhantes de adultos face ao processo de hospitalização. Escola Anna Nery, 16(1), 134-140. Recuperado em março 8, 2016, de http://www. scielo.br/scielo. $p h p$ ?script=sci_arttext\&pid=S1414$81452012000100018 \& \operatorname{lng}=e n \&$ tIng=pt

Conselho Nacional de Saúde (Brasil). (1996). Resolução normativa $n^{\circ} 196$, de 1996. Aprova diretrizes e normas regulamentadoras de pesquisa envolvendo seres humanos. Brasília: Ministério da Saúde.

Colesante, M. F. L., Gomes, I. P., Morais, J. D., \& Collet, N. (2012). Impacto na vida de mães cuidadoras de crianças com doença crônica. Revista de Enfermagem UERJ, 23(4), 501-506. Recuperado em março 10, 2016, de http://www.e-publicacoes.uerj.br/index.php/ enfermagemuerj/article/view/4966/14241

Costa, J. B., Mombelli, M. A., \& Marcon, S. S. (2009). Avaliação do sofrimento psíquico da mãe acompanhante em alojamento conjunto pediátrico. Estudos de Psicologia (Campinas), 26(3), 317-325. https://doi. org/10.1590/S0103-166X2009000300005

Del Bianco Faria, A. M., \& Cardoso, C. L. (2010). Aspectos psicossociais de acompanhantes cuidadores de crianças com câncer: stress e enfrentamento. Estudos de Psicologia (Campinas), 27(1), 13-20. https://doi. org/10.1590/S0103-166X2010000100002

Faria, J. B., \& Seidl, E. M. F. (2005). Religiosidade e enfrentamento em contextos de saúde e doença: revisão de literatura. Psicologia: Reflexão e Crítica, 18(3), 381389. Recuperado em dezembro 22, 2015, de http:// www.scielo.br/pdf/prc/v18n3/a12v18n3.pdf

Farinhas, G. V., Wendling, M. I., \& Dellazzana-Zanon, L. L. (2013). Impacto psicológico do diagnóstico de câncer na família: um estudo de caso a partir da percepção do cuidador. Pensando Famílias, 17(2), 111-129. Recuperado em março 8, 2016, de http://pepsic.bvsalud. org/scielo.php?script=sci_arttext\&pid=\$1679-494X20 13000200009\&lng=pt\&tlng=pt

Fornazari, S. A., \& Ferreira, R. E. R. (2010). Religiosidade/ espiritualidade em pacientes oncológicos: qualidade de vida e saúde. Psicologia: Teoria e Pesquisa, 26(2), 265-272. Recuperado em dezembro 22, 2015, de http://www.scielo.br/scielo. php?script=sci_arttext $\&$ pid $=$ S0102-377220100002 00008\&lng=en\&ting=pt

Fujimura, M., Sonobe, S., Nishijima, Y., Niizuma, K., Sakata, H., Kure, S., \& Tominaga, T. (2014). Genetics and biomarkers of Moyamoya disease: Significance of RNF213 as a susceptibility gene. Journal of Stroke, 16(2), 65-72. Retrieved March 8, 2016, from http://www.ncbi. nlm.nih.gov/pubmed/ 24949311

Furtado, M. C. C., \& Lima, R. A. G. (2003). O cotidiano da família com filhos portadores de fibrose cística: subsídios para a enfermagem pediátrica. Revista Latino-Americana de Enfermagem, 11(1), 66-73. Recuperado em dezembro 28, 2015, de http://www. scielo.br/scielo.php? script=sci_arttext\&pid=S010411692003000100010\&lng=en\&tlng $=p t$

Horta, C. R., Neme, C. M. B., Capote, P. S. O., \& Gibran, V. M. (2003). O papel da fé no enfrentamento do câncer. In C. M. B. Neme \& O. M. P. R. Rodrigues (Orgs.), Psicologia da saúde: perspectivas interdisciplinares (pp.149-172). São Carlos: Rima.

Hsu, Y. H., Kuo, M. F., Hua, M. S., \& Yang, C. C. (2014). Selective neuropsychological impairments and related clinical factors in children with Moyamoya disease of the 
transient ischemic attack type. Child's Nervous System, 30(3), 441-447. Retrieved July 15, 2015, from http://link. springer.com/article/10.1007\%2Fs00381-013-2271-9

Junqueira, P. A., \& Moura-Ribeiro, M. V. L. (2002). Síndrome de Down e Moyamoya: estudo através de metanálise. Arquivos de Neuropsiquiatria, 60(2-A), 274-280. Recuperado em junho 22, 2015, de http://www.scielo. br/pdf/anp/v60n2A/a17v60n2.pdf

Kainth, D. S., Chaudhry, S. A., Kainth, H. S., Suri, F. K., \& Qureshi, A. I. (2013). Prevalence and characteristics of concurrent Down syndrome in patients with Moyamoya disease. Neurosurgery, 72(2), 210-215. Retrieved May 12, 2015, from http://www.ncbi.nlm.nih.gov/ pubmed/23149966

Kazumata, K., Tha, K. K., Narita, H., Kusumi, I., Shichinohe, H., Ito, M., ... Houkin, K. (2015). Chronic ischemia alters brain microstructural integrity and cognitive performance in adult Moyamoya disease. Journal of Stroke, 46(2), 354-360. Retrieved March 7, 2016, from http://www.ncbi.nlm.nih.gov/pubmed/25538200

Kim, J. S. (2016). Moyamoya disease: Epidemiology, clinical features, and diagnosis. Journal of Stroke, 18(1), 2-11. Retrieved March 7, 2016, from http://www.ncbi.nlm. nih.gov/pubmed/26846755

Kim, T., Oh, C. W., Bang, J. S., Kim, J. E., \& Cho, W. S. (2016). Moyamoya disease: Treatment and outcomes. Journal of Stroke, 18(1), 21-30. Retrieved March 7, 2016, from http://www.ncbi.nlm.nih.gov/pubmed/26846757

Kim, T., Oh, C. W., Kwon, O. K., Hwang, G., Kim, J. E., Kang, H. S., ... Bang, J. S. (2015). Stroke prevention by direct revascularization for patients with adultonset Moyamoya disease presenting with ischemia. Journal of Neurosurgery, 124(6), 1788-1793. Retrieved March 8, 2016, from http://www.ncbi.nlm.nih.gov/ pubmed/26636391

Matushita, H., Matushita, T. T., Cardeal, D., \& Andrade, F. (2014). Doenças cérebro-vasculares na infância. Revista Brasileira de Neurologia e Psiquiatria, 18(2), 165-175. Recuperado em março 11, 2016 de http://www. revneuropsiq.com.br/rbnp/article/view/84/46

Miyamoto, S., Yoshimoto, T., Hashimoto, N., Okada, Y., Tsuji, I., Tominaga, T., ... Takahashi, J. C. (2014). Effects of extracranial-intracranial bypass for patients with hemorrhagic Moyamoya disease: Results of the Japan adult Moyamoya trial. Journal of Stroke, 45(5), 14151421. Retrieved March 8, 2016 from http://www.ncbi. nlm.nih.gov/pubmed/24668203

Nakamizo, A., Kikkawa, Y., Hiwatashi, A., Matsushima, T., \& Sasaki, T. (2014). Executive function and diffusion in frontal white matter of adults with Moyamoya disease. Journal of Stroke and Cerebrovascular Diseases, 23(3), 457-431. Retrieved July 21, 2015, from http://www. ncbi.nlm.nih.gov/pubmed/23608368
Nascimento, T. L., Santos, A. J., Forbrig, J. C., Soder, R. B., Martins, R. T., Cunha, R. S., \& D'Almeida, A. C. G. (2011). Doença de Moyamoya: uma abordagem diagnóstica e o tratamento cirúrgico. Revista da AMRIGS, 55(3), 277-281. Recuperado em junho 15, 2015, de http://www.amrigs.com.br/revista/5503/0000045956-Revista_AMRIGS_3_relato_de_caso_ doena_de_moyamoya.pdf

Neme, C. M. B., \& Lipp, M. E. N. (2010). Estresse psicológico e enfrentamento em mulheres com e sem câncer. Psicologia: Teoria e Pesquisa, 26(3), 475-483. Recuperado em junho 22, 2015, de http://www.scielo.br/scielo.php? script=sci_arttext\&pid=S0102-37722010000300010

Neme, C. M. B. (2005). Ganhos terapêuticos com psicoterapia breve em serviço de psico-oncologia hospitalar. In C. P. Simon, L. L. Melo-Silva, \& M. A. Santos (Eds.), Formação em psicologia: desafios da diversidade na pesquisa e na prática (pp.39-68). São Paulo: Vetor.

Neme, C. M. B. (2012) Doenças crônicas: fatores de risco e mecanismos de proteção no câncer infantil. In L. E. Melchiori, O. M. P. R. Rodrigues, \& A. C. B. Maia. Famílias e crianças: reflexões teórico-práticas sobre os cuidados com as crianças (pp.105-118). Curitiba: Juruá Psicologia.

Nieweglowski, V. H., \& Moré, C. L. O. O. (2008). Comunicação equipe-família em unidade de terapia intensiva pediátrica: impacto no processo de hospitalização. Estudos de Psicologia (Campinas), 25(1), 111-122. https://doi.org/10.1590/S0103-166X2008000100011

Nóbrega, V. M., Reichert, A. P. S., Silva, K. L., Coutinho, S. E. D., \& Collet, N. (2012). Imposições e conflitos no cotidiano das famílias de crianças com doença crônica. Escola Anna Nery, 16(4), 781-788. Recuperado em março 10, 2016, de http://www.scielo.br/scielo.php?script=sci_ arttext\&pid=S1414-81452012000400020\&lng=en\& tlng=pt

Oliveira, M. R., \& Junges, J. R. (2012). Saúde mental e espiritualidade/religiosidade: a visão de psicólogos. Estudos de Psicologia (Natal), 17(3), 469-476. Recuperado em março 12, 2016, de http://www. scielo.br/scielo. php?script=sci_arttext\&pid=\$1413294X2012000300016

Ribeiro, N. R. R. (1999). Famílias vivenciando o risco de vida do filho (Tese de doutorado não-publicada). Universidade Federal de Santa Catarina, Florianópolis. Recuperado em junho 22, 2015, de http://www.lume. ufrgs.br/handle/10183/2936?locale=pt_BR

Rooke, M. I., \& Pereira-Silva, N. L. (2016). Indicativos de resiliência familiar em famílias de crianças com síndrome de Down. Estudos de Psicologia (Campinas), 33(1), 117-126. https://doi.org/10.1590/1982027520160001000012

Ruiz, V. H., \& Pulgarin, J. A. H. (2012). Enfermedad de Moyamoya: Reporte de caso y revisión de la literatura. 
Revista Médica de Risaralda, 18(2), 172-176. Recuperado en Mayo 11, 2012, de http://www.scielo.org.co/scielo. php?pid=S0122-06672012000200012\&script $=$ sci_ arttext

Santos, A. F. O., \& Cardoso, C. L. (2015). Family members of individuals suffering from mental disorders: Stress and care stressors. Estudos de Psicologia (Campinas), 32(1), 87-95. Recuperado em julho 30, 2015, de http://www.scielo.br/scielo.php?pid=S0103166X2015000100087\&script=sci_arttext

Santos, L. M. P., \& Gonçalves, L. L. C. (2008). Crianças com câncer: desvelando o significado do adoecimento atribuído por sua mãe. Revista de Enfermagem UERJ, 16(2), 224-229. Recuperado em julho 30, 2015, de http://www.facenf.uerj.br/v16n2/v16n2a14.pdf

Shoukat, S., Itrat, A., Taqui, A. M., Zaidi, M., \& Kamal, A. K. (2009). Moyamoya disease: A clinical spectrum, literature review and case series from a tertiary care hospital in Paskitan. BMC Neurology, 9(15), 1-8. Retrieved December 22, 2015, from http://www.ncbi. nlm.nih.gov/pubmed/19368734
Silva, L. C., Weiss, E. M., Bernardes, D. B., \& Souza, A. I. J. (2006). Hospitalização e morte na infância: desafios das famílias. Família, Saúde e Desenvolvimento, 1(8), 73-79. Recuperado em junho 22, 2015, de http://ojs.c3sl.ufpr. br/ojs/index.php/refased/article/view/8024

Su, S. H., Haj, J., Zhang, L., Wu, Y. F., \& Yu, F. (2013). Quality of life and psychological impact in adult patients with hemorrhagic Moyamoya disease who received no surgical revascularization. Journal of the Neurological Sciences, 328(1-2), 32-36. Retrieved June 22, 2015, from https://www.ncbi.nlm.nih.gov/pubmed/23498370

Tabaquim, M. L. M., \& Marquesini, M. A. M. (2013). Study of the stress of parents of patients with cleft lip and palate in a surgical process. Estudos de Psicologia (Campinas), 30(4), 517-524. https://doi.org/10.1590/ S0103-166X2013000400005

Recebido: agosto 27, 2015

Versão final: março 18, 2016

Aprovado: abril 8, 2016 